\title{
Design sensor-less PID filter controller for first order delays system
}

\begin{abstract}
The dynamics of a first order delay system is highly nonlinear, time variant, uncertain and coupling effects. The main objectives to control of first order delay system are time response and acceleration measurements. The problem of acceleration measurements can be reduced, based on design sensor-less Proportional-Integral-Derivative (PID) filter controller in this research. Assuming unstructured uncertainties and structure uncertainties can be defined into one term and considered as an uncertainty and external disturbance, the problem of computation burden and large number of parameters can be solved to some extent. To solve the uncertainties acceleration measurements play an important role. In order to design sensorless PID filter controller, an accurate PD surface and the derivative of PD surface plays important role. To design an accurate PD surface, stable and tuning surface slope is needed to form the structure of main PID controller. In this algorithm, the derivative of PD surface computes the second derivation of error. Regarding to this method, the challenge of system uncertainties and time response have been solved based on sensor-less acceleration linear filter controller. As this point if $\mathrm{s}=\mathrm{K} 1 \mathrm{e}+\mathrm{e}+\mathrm{K} 2 \mathrm{E} \mathrm{e}$ is chosen as desired surface, if the dynamic of first order delay is derived to surface then the linearization can be realized. Because, when the system dynamic is on the surface is used the derivative of surface $S=K 1 \mathrm{e}$ $+\mathrm{e}+\mathrm{K} 2 \mathrm{e}$ is equal to the zero that is a decoupled and linearized closed-loop systems dynamics. Linearization and decoupling by the above method can be obtained in spite of the quality of the first order delay dynamic model.
\end{abstract}

Keyword: First order delays system; Position PID controller; Sensor-less control algorithm; PD surface algorithm 